\title{
A New Approach to Constructing Tolerance Limits on Order Statistics in Future Samples Coming from a Normal Distribution
}

\author{
${ }^{1}$ Nicholas A. Nechval, ${ }^{2}$ Konstantin N. Nechval, and ${ }^{3}$ Vladimir F. Strelchonok \\ ${ }^{1,3}$ Department of Mathematics, Baltic International Academy, Riga, Latvia; \\ ${ }^{2}$ Department of Applied Mathematics, Transport and Telecommunication Institute, Riga, Latvia; \\ nechval@junik.Iv; konstan@tsi.lv; str_riga@inbox.lv
}

\begin{abstract}
Although the concept of statistical tolerance limits has been well recognized for long time, surprisingly, it seems that their applications remain still limited. Analytic formulas for the tolerance limits are available in only simple cases, for example, for the upper or lower tolerance limit for a univariate normal population. Thus it becomes necessary to use new or innovative approaches which will allow one to construct tolerance limits on future order statistics for many populations. In this paper, a new approach to constructing lower and upper tolerance limits on order statistics in future samples is proposed. Attention is restricted to invariant families of distributions under parametric uncertainty. The approach used here emphasizes pivotal quantities relevant for obtaining tolerance factors and is applicable whenever the statistical problem is invariant under a group of transformations that acts transitively on the parameter space. It does not require the construction of any tables and is applicable whether the past data are complete or Type II censored. The proposed approach requires a quantile of the $F$ distribution and is conceptually simple and easy to use. For illustration, the normal distribution is considered. The discussion is restricted to one-sided tolerance limits. A practical example of finding a warranty assessment of image quality is given.
\end{abstract}

Keywords: Order Statistics, F Distribution, Lower Tolerance Limit, Upper Tolerance Limit, Normal Distribution.

\section{Introduction}

Statistical tolerance limits are an important tool often utilized in areas such as engineering, manufacturing, and quality control for making statistical inference on an unknown population. As opposed to a confidence limit that provides information concerning an unknown population parameter, a tolerance limit provides information on the entire population; to be specific, one-sided tolerance limit is expected to capture a certain proportion or more of the population, with a given confidence level. For example, an upper tolerance limit for a univariate population is such that with a given confidence level, a specified proportion or more of the population will fall below the limit. A lower tolerance limit satisfies similar conditions.

It is often desirable to have statistical tolerance limits available for the distributions used to describe time-to-failure data in reliability problems. For example, one might wish to know if at least a certain proportion, say $\beta$, of a manufactured product will operate at least $T$ hours. This question can not usually be answered exactly, but it may be possible to determine a lower tolerance limit $L\left(X_{1}, \ldots, X_{n}\right)$, 
based on a preliminary random sample $\left(X_{1}, \ldots, X_{n}\right)$, such that one can say with a certain confidence $\gamma$ that at least $100 \beta \%$ of the product will operate longer than $L\left(X_{1}, \ldots, X_{n}\right)$. Then reliability statements can be made based on $L\left(X_{1}, \ldots, X_{n}\right)$, or, decisions can be reached by comparing $L\left(X_{1}, \ldots, X_{n}\right)$ to $T$. Tolerance limits of the type mentioned above are considered in this paper. That is, if $f_{\theta}(x)$ denotes the density function of the parent population under consideration and if $S$ is any statistic obtained from the preliminary random sample $\left(X_{1}, \ldots, X_{n}\right)$ of that population, then $L(S)$ is a lower $\gamma$ probability tolerance limit for proportion $\beta$ if

$$
\operatorname{Pr}\left(\int_{L(S)}^{\infty} f_{\theta}(x) d x \geq \beta\right)=\gamma,
$$

and $U(S)$ is an upper $\gamma$ probability tolerance limit for proportion $\beta$ if

$$
\operatorname{Pr}\left(\int_{-\infty}^{U(S)} f_{\theta}(x) d x \geq \beta\right)=\gamma,
$$

where $\theta$ is the parameter (in general, vector).

The common distributions used in life testing problems are the normal, exponential, Weibull, and gamma distributions [1]. Tolerance limits for the normal distribution have been considered in [2], [3], [4], and others.

Tolerance limits enjoy a fairly rich history in the literature and have a very important role in engineering and manufacturing applications. Patel [5] provides a review (which was fairly comprehensive at the time of publication) of tolerance limits for many distributions as well as a discussion of their relation with confidence intervals for percentiles and prediction intervals. Dunsmore [6] and Guenther, Patil, and Uppuluri [7] both discuss 2-parameter exponential tolerance intervals and the estimation procedure in greater detail. Engelhardt and Bain [8] discuss how to modify the formulas when dealing with type II censored data. Guenther [9] and Hahn and Meeker [10] discuss how one-sided tolerance limits can be used to obtain approximate two-sided tolerance intervals by applying Bonferroni's inequality. Tolerance limits on order statistics in future samples coming from a two-parameter exponential distribution have been considered in [11].

In contrast to other statistical limits commonly used for statistical inference, the tolerance limits (especially for the order statistics) are used relatively rarely. One reason is that the theoretical concept and computational complexity of the tolerance limits is significantly more difficult than that of the standard confidence and prediction limits. Thus it becomes necessary to use new or innovative approaches which will allow one to construct tolerance limits on future order statistics for many populations.

In this paper, a new approach to constructing lower and upper tolerance limits on order statistics in future samples is proposed. For illustration, the normal distribution is considered. It is a commonly used model in reliability and risk theory. Although the concept of statistical tolerance limits has been well recognized for long time, surprisingly, it seems that their applications remain still limited. 


\section{Mathematical Preliminaries}

\subsection{Probability Distribution Function of Order Statistic}

Theorem 1. If there is a random sample of $m$ ordered observations $Y_{1} \leq \ldots \leq Y_{m}$ from a known distribution (continuous or discrete) with density function $f_{\theta}(y)$, distribution function $F_{\theta}(y)$, then the probability distribution function of the $k$ th order statistic $Y_{k}, k \in\{1,2, \ldots, m\}$, is given by

$$
P_{\theta}\left(Y_{k} \leq y_{k} \mid m\right)=\int_{\frac{1-F_{\theta}\left(y_{k}\right)}{F_{\theta}\left(y_{k}\right)}}^{\infty} f_{2(m-k+1), 2 k}(x) d x,
$$

where

$$
\begin{aligned}
f_{2(m-k+1), 2 k}(x)= & \frac{1}{\mathrm{~B}\left(\frac{2(m-k+1)}{2}, \frac{2 k}{2}\right)}\left(\frac{2(m-k+1)}{2 k}\right)\left(\frac{2(m-k+1)}{2 k} x\right)^{2(m-k+1) / 2-1} \\
& \times\left(1+\frac{2(m-k+1)}{2 k} x\right)^{-[2(m-k+1)+2 k] / 2}, \quad x>0,
\end{aligned}
$$

is the probability density function of an $F$ distribution with $2(m-k+1)$ and $2 k$ degrees of freedom.

Proof. Suppose an event occurs with probability $p$ per trial. It is well-known that the probability $P$ of its occurring $k$ or more times in $m$ trials is termed a cumulative binomial probability, and is related to the incomplete beta function $I_{x}(a, b)$ as follows:

$$
P \equiv \sum_{j=k}^{m}\left(\begin{array}{c}
m \\
j
\end{array}\right) p^{j}(1-p)^{m-j}=I_{p}(k, m-k+1) .
$$

It follows from (5) that

$$
\begin{gathered}
P_{\theta}\left\{Y_{k} \leq y_{k} \mid m\right\}=\sum_{j=k}^{m}\left(\begin{array}{c}
m \\
j
\end{array}\right)\left[F_{\theta}\left(y_{k}\right)\right]^{j}\left[1-F_{\theta}\left(y_{k}\right)\right]^{m-j}=I_{F_{\theta}\left(y_{k}\right)}(k, m-k+1) \\
=\frac{1}{\mathrm{~B}(k, m-k+1)} \int_{0}^{F_{\theta}\left(y_{k}\right)} u^{k-1}(1-u)^{(m-k+1)-1} d u=\frac{\left(\frac{2(m-k+1)}{2 k}\right)^{2(m-k+1) / 2}}{\mathrm{~B}\left(\frac{2 k}{2}, \frac{2(m-k+1)}{2}\right)} \int_{0}^{F_{\theta}\left(y_{k}\right)} u^{\frac{2(m-k+1)+2 k}{2}} \\
\times\left(\frac{1-u}{u} \frac{2 k}{2(m-k+1)}\right)^{2(m-k+1) / 2-1} \frac{-2 k}{2(m-k+1)}\left(-\frac{d u}{u^{2}}\right) \\
=\frac{\left(\frac{2(m-k+1)}{2 k}\right)^{2(m-k+1) / 2}}{\mathrm{~B}\left(\frac{2(m-k+1)}{2}, \frac{2 k}{2}\right)} \int_{\left.\frac{1-F_{\theta}\left(y_{k}\right)}{F_{\theta}\left(y_{k}\right)}\right)_{2 k}^{\infty(m-k+1)}}^{\infty} x^{2(m-k+1) / 2-1}\left(1+\frac{2(m-k+1)}{2 k} x\right)^{-[2(m-k+1)+2 k] / 2} d x,
\end{gathered}
$$

where 


$$
x=\frac{1-u}{u} \frac{2 k}{2(m-k+1)} .
$$

This ends the proof.

Corollary 1.1

$$
P_{\theta}\left(Y_{k}>y_{k} \mid m\right)=1-P_{\theta}\left\{Y_{k} \leq y_{k} \mid m\right\}=\int_{0}^{\frac{1-F_{\theta}\left(y_{k}\right)}{F_{\theta}\left(y_{k}\right)}} \int_{2(m-k+1), 2 k}^{\frac{2 k}{2(m-k+1)}}(x) d x .
$$

Corollary 1.2. If $y_{k, m ; \gamma}$ is the quantile of order $\gamma$ for the distribution of $Y_{k}$, we have from (8) that $y_{k, m ; \gamma}$ is the solution of

$$
F_{\theta}\left(y_{k, m ; \gamma}\right)=\frac{k}{k+(m-k+1) q_{2(m-k+1), 2 k ; 1-\gamma}},
$$

where $q_{2(m-k+1), 2 k ; 1-\gamma}$ is the quantile of order $1-\gamma$ for the $F$ distribution with $2(m-k+1)$ and $2 k$ degrees of freedom.

\subsection{Normal Distribution}

The normal distribution is perhaps the most commonly used probability distribution in both statistical theory and applications. For example: 1) many classical statistical tests are based on the assumption that the data follow a normal distribution (this assumption should be tested before applying these tests); 2) in modeling applications, such as linear and non-linear regression, the error term is often assumed to follow a normal distribution with fixed location and scale; 3) the normal distribution is used to find significance levels in many hypothesis tests and confidence intervals.

Physical measurements in areas such as meteorological experiments, rainfall studies, and measurements of manufactured parts are often more than adequately explained with a normal distribution. In addition, errors in scientific measurements are extremely well approximated by a normal distribution.

In 1733, Abraham DeMoivre developed the mathematical equation of the normal curve. It provided a basis for which much of the theory of inductive statistics is founded. The normal distribution is often referred to as the Gaussian distribution, in honor of Karl Gauss (1777-1855), who also derived its equation from a study of errors in repeated measurements of the same quantity. Properties of the normal distribution have been well developed (e.g., see Johnson et al. [12], Patel and Read [13], Balakrishnan and Nevzorov \{14], Kotz and Vicari [15].

The normal distribution plays a vital role in many applied problems of biology, economics, engineering, financial risk management, genetics, hydrology, mechanics, medicine, number theory, statistics, physics, psychology, reliability, etc., and has been extensively studied, both from theoretical and applications point of view, by many researchers, since its inception.

Thus, the normal distribution is a widely used and widely known distribution. It is characterized by the probability density function of a continuous random variable $Y$,

$$
f_{\theta}(y)=\frac{1}{\sigma \sqrt{2 \pi}} \exp \left(-\frac{(y-\mu)^{2}}{2 \sigma^{2}}\right), \quad-\infty<y<\infty,
$$


that is, $Y \sim N\left(\mu, \sigma^{2}\right)$, where $\theta=(\mu, \sigma),-\infty<\mu<\infty$ is the location parameter and $\sigma>0$ is the scale parameter. These parameters are assumed to be unknown. The cumulative distribution function of the normal distribution is given by

$$
F_{\theta}(y)=\frac{1}{\sigma \sqrt{2 \pi}} \int_{-\infty}^{y} \exp \left(-\frac{(y-\mu)^{2}}{2 \sigma^{2}}\right) d y .
$$

It is known (Nechval and Vasermanis [16]) that the complete sufficient statistic for the parameter $\theta$, based on observations in a random sample $\left(X_{1}, \ldots, X_{n}\right)$ of size $n$ from the normal distribution (10) is given by

$$
S=\left(\bar{X}=\sum_{i=1}^{n} X_{i} / n, S_{1}^{2}=\sum_{i=1}^{n}\left(X_{i}-\bar{X}\right)^{2} /(n-1)\right) .
$$

Here the following theorem takes place.

Theorem 2. Let $\left(X_{1}, \ldots, X_{n}\right)$ be a preliminary random sample from the normal distribution (10) , where it is assumed that the parameter $\theta=(\mu, \sigma)$ is unknown. Then the joint probability density function of the pivotal quantities,

$$
V_{1}=\frac{\sqrt{n}(\bar{X}-\mu)}{\sigma}, \quad V_{2}=\frac{(n-1) S_{1}^{2}}{\sigma^{2}},
$$

is given by

$$
f(v)=f_{1}\left(v_{1}\right) f_{2}\left(v_{2}\right),
$$

where

$$
\begin{gathered}
V=\left(V_{1}, V_{2}\right), \\
f_{1}\left(v_{1}\right)=\frac{1}{\sqrt{2 \pi}} \exp \left(-\frac{v_{1}^{2}}{2}\right), \quad-\infty<v_{1}<\infty, \\
f_{2}\left(v_{2}\right)=\frac{1}{2^{(n-1) / 2} \Gamma((n-1) / 2)} v_{2}^{(n-1) / 2-1} \exp \left(-v_{2} / 2\right), \quad v_{2} \geq 0 .
\end{gathered}
$$

Proof. The joint density of $X_{1}, \ldots, X_{n}$ is given by

$$
\begin{aligned}
f_{\theta}\left(x_{1}, \ldots, x_{n}\right) & =\prod_{i=1}^{r} f_{\theta}\left(x_{i}\right)=\prod_{i=1}^{n}\left(2 \pi \sigma^{2}\right)^{-1 / 2} \exp \left(-\frac{1}{2 \sigma^{2}}\left(x_{i}-\mu\right)^{2}\right) \\
= & \left(2 \pi \sigma^{2}\right)^{-n / 2} \exp \left(-\frac{1}{2 \sigma^{2}} \sum_{i=1}^{n}\left(x_{i}-\mu\right)^{2}\right) .
\end{aligned}
$$

Using the invariant embedding technique [17]-[19], we transform (18) to

$$
\begin{gathered}
f_{\theta}\left(x_{1}, \ldots, x_{n}\right) d \bar{x} d s_{1}^{2}=\left(2 \pi \sigma^{2}\right)^{-n / 2} \exp \left(-\frac{1}{2 \sigma^{2}} \sum_{i=1}^{n}\left(x_{i}-\bar{x}+\bar{x}-\mu\right)^{2}\right) d \bar{x} d s_{1}^{2} \\
=\left(2 \pi \sigma^{2}\right)^{-n / 2} \exp \left(-\frac{1}{2 \sigma^{2}} \sum_{i=1}^{n}\left[\left(x_{i}-\bar{x}\right)^{2}+2\left(x_{i}-\bar{x}\right)(\bar{x}-\mu)+(\bar{x}-\mu)^{2}\right]\right) d \bar{x} d s_{1}^{2}
\end{gathered}
$$




$$
\begin{gathered}
=\left(2 \pi \sigma^{2}\right)^{-n / 2} \exp \left(-\frac{1}{2 \sigma^{2}}\left[\sum_{i=1}^{n}\left(x_{i}-\bar{x}\right)^{2}+2(\bar{x}-\mu) \sum_{i=1}^{n}\left(x_{i}-\bar{x}\right)+n(\bar{x}-\mu)^{2}\right]\right) d \bar{x} d s_{1}^{2} \\
=\left(2 \pi \sigma^{2}\right)^{-n / 2} \exp \left(-\frac{1}{2 \sigma^{2}}\left[\sum_{i=1}^{n}\left(x_{i}-\bar{x}\right)^{2}+n(\bar{x}-\mu)^{2}\right]\right) d \bar{x} d s_{1}^{2} \\
=n^{-1 / 2}(2 \pi)^{-1 / 2} \exp \left(-\frac{n(\bar{x}-\mu)^{2}}{2 \sigma^{2}}\right) d\left(\frac{\sqrt{n}(\bar{x}-\mu)}{\sigma}\right) \\
\times(\pi)^{-(n-1) / 2}(n-1)^{-(n-1) / 2}\left(s_{1}^{2}\right)^{-(n-1) / 2}\left(\frac{(n-1) s_{1}^{2}}{2 \sigma^{2}}\right)^{(n-1) / 2-1} \exp \left(-\frac{(n-1) s_{1}^{2}}{2 \sigma^{2}}\right) d\left(\frac{(n-1) s_{1}^{2}}{2 \sigma^{2}}\right) \\
\propto\left(\frac{v_{2}}{2}\right)^{(n-1) / 2-1} \exp \left(-\frac{v_{2}}{2}\right) d\left(\frac{v_{2}}{2}\right) .
\end{gathered}
$$

Normalizing (19), we obtain (14). This ends the proof.

Thus,

$$
V_{1} \sim N(0,1), \quad V_{2} \sim \chi_{n-1}^{2},
$$

where $V_{2}$ is statistically independent of $V_{1}$.

Corollary 2.1. If $V_{1}$ is a normally distributed random variable with unit variance and zero mean, and $V_{2}$ is a chi-squared distributed random variable with $n-1$ degrees of freedom that is statistically independent of $V_{1}$, then

$$
T=\frac{V_{1}+\Delta}{\sqrt{V_{2} /(n-1)}}=\frac{V_{1}+\Delta}{\sqrt{W}} \sim f_{n-1, \Delta}(t), \quad-\infty<t<\infty,
$$

is a non-central $t$-distributed random variable with $n-1$ degrees of freedom and non-centrality parameter $\Delta$, where

$$
\begin{gathered}
W=\frac{V_{2}}{n-1}=\frac{S_{1}^{2}}{\sigma^{2}} \sim f_{n-1}(w)=\frac{(n-1)^{(n-1) / 2}}{2^{(n-1) / 2} \Gamma((n-1) / 2)} w^{(n-1) / 2-1} \exp (-(n-1) w / 2), \quad w \geq 0, \\
f_{n-1, \Delta}(t)=\frac{(n-1)^{(n-1) / 2}}{\sqrt{\pi} \Gamma((n-1) / 2) 2^{n / 2}} \frac{\exp \left(-\frac{(n-1) \Delta^{2}}{2\left(t^{2}+n-1\right)}\right)}{\left(t^{2}+n-1\right)^{n / 2}} \\
\quad \times \int_{0}^{\infty} w_{\bullet}^{n / 2-1} \exp \left(-\frac{1}{2}\left[w_{\bullet}^{1 / 2}-\frac{t \Delta}{\sqrt{t^{2}+n-1}}\right]^{2}\right) d w_{\bullet}, \quad-\infty<t<\infty,
\end{gathered}
$$

is the probability density function of $T$, 


$$
F_{n-1, \Delta}(t)=\operatorname{Pr}(T \leq t)=\frac{(n-1)^{(n-1) / 2}}{2^{(n-1) / 2} \Gamma((n-1) / 2)} \int_{0}^{\infty} w^{(n-1) / 2-1} \exp (-(n-1) w / 2) \Phi(t \sqrt{w}-\Delta) d w,
$$

is the cumulative distribution function of $T . \Phi(x)$ is the standard normal distribution function. Note that the non-centrality parameter $\Delta$ may be negative.

\section{Tolerance Limits on Order Statistic}

\subsection{Lower Tolerance Limit}

Theorem 3. Let $X_{1}, \ldots, X_{n}$ be observations from a preliminary sample of size $n$ from a normal distribution defined by the probability density function (10). Then a lower one-sided $\beta$-content tolerance limit at level $\gamma_{1} L_{k} \equiv L_{k}(S)$ (on the $k$ th order statistic $Y_{k}$ from a set of $m$ future ordered observations $Y_{1} \leq \ldots \leq Y_{m}$ also from the distribution (10)), which satisfies

$$
\operatorname{Pr} P_{\theta}\left(Y_{k}>L_{k} \mid m\right) \geq \beta=\gamma
$$

is given by

$$
L_{k}=\bar{X}+\eta_{L} S_{1}
$$

where

$$
\eta_{L}=-\frac{t_{r, \Delta ; \gamma}}{\sqrt{n}}
$$

is the lower tolerance factor, $t_{r, \Delta ; \gamma}$ is the quantile of order $\gamma$ for the non-central $t$-distribution with $r=n-1$ degrees of freedom and non-centrality parameter $\Delta=-z_{1-\delta_{\beta}} \sqrt{n}, \quad z_{1-\delta_{\beta}}$ denotes the $1-\delta_{\beta}$ quantile of a standard normal distribution,

$$
\delta_{\beta}=\frac{(m-k+1) q_{2(m-k+1), 2 k ; \beta}}{(m-k+1) q_{2(m-k+1), 2 k ; \beta}+k},
$$

$q_{2(m-k+1), 2 k ; \beta}$ is the quantile of order $\beta$ for the $F$ distribution with $2(m-k+1)$ and $2 k$ degrees of freedom.

Proof. It follows from (8), (11) and (25) that

$$
\begin{gathered}
\operatorname{Pr} P_{\theta}\left(Y_{k}>L_{k} \mid m\right) \geq \beta=\operatorname{Pr}\left(\int_{0}^{\left.\frac{1-F_{\theta}\left(L_{k}\right)}{F_{\theta}\left(L_{k}\right)} f_{2(m-k+1), 2 k}^{\frac{2 k}{2(m-k+1)}}(x) d x \geq \beta\right)}\right. \\
=\operatorname{Pr}\left(\frac{1-F_{\theta}\left(L_{k}\right)}{F_{\theta}\left(L_{k}\right)} \frac{2 k}{2(m-k+1)} \geq q_{2(m-k+1), 2 k ; \beta}\right)=\operatorname{Pr}\left(F_{\theta}\left(L_{k}\right) \leq \frac{k}{k+(m-k+1) q_{2(m-k+1), 2 k ; \beta}}\right) \\
=\operatorname{Pr}\left(\frac{1}{\sigma \sqrt{2 \pi}} \int_{L_{k}}^{\infty} \exp \left(-\frac{(y-\mu)^{2}}{2 \sigma^{2}}\right) d y \geq \frac{(m-k+1) q_{2(m-k+1), 2 k ; \beta}}{(m-k+1) q_{2(m-k+1), 2 k ; \beta}+k}\right)
\end{gathered}
$$




$$
\begin{gathered}
=\operatorname{Pr}\left(\frac{1}{\sqrt{2 \pi}} \int_{\frac{L_{k}-\mu}{\sigma}}^{\infty} \exp \left(-\frac{z^{2}}{2}\right) d z \geq \delta_{\beta}\right)=\operatorname{Pr}\left(\frac{1}{\sqrt{2 \pi}} \int_{\infty}^{\frac{L_{k}-\mu}{\sigma}} \exp \left(-\frac{z^{2}}{2}\right) d z \leq 1-\delta_{\beta}\right) \\
=\operatorname{Pr}\left(\frac{L_{k}-\mu}{\sigma} \leq z_{1-\delta_{\beta}}\right)=\operatorname{Pr}\left(\frac{L_{k}-\bar{X}+\bar{X}-\mu}{\sigma} \leq z_{1-\delta_{\beta}}\right) \\
=\operatorname{Pr}\left(\frac{L_{k}-\bar{X}}{S_{1}} \sqrt{n} \frac{S_{1}}{\sigma}+\frac{\sqrt{n}(\bar{X}-\mu)}{\sigma} \leq z_{1-\delta_{\beta}} \sqrt{n}\right)=\operatorname{Pr}\left(\eta_{L} \sqrt{n} \sqrt{W}+V_{1} \leq z_{1-\delta_{\beta}} \sqrt{n}\right) \\
=\operatorname{Pr}\left(\frac{V_{1}-z_{1-\delta_{\beta}} \sqrt{n}}{\sqrt{W}} \leq-\eta_{L} \sqrt{n}\right)=\operatorname{Pr}\left(\frac{V_{1}+\Delta}{\sqrt{W}} \leq-\eta_{L} \sqrt{n}\right)=\operatorname{Pr}\left(T \leq-\eta_{L} \sqrt{n}\right)=F_{r, \Delta}(t),
\end{gathered}
$$

where

$$
\eta_{L}=\frac{L_{k}-\bar{X}}{S_{1}}
$$

is the lower tolerance factor,

$$
\Delta=-z_{1-\delta_{\beta}} \sqrt{n}, \quad r=n-1, \quad t=-\eta_{L} \sqrt{n} .
$$

It follows from (25), (29) and (31) that the lower tolerance factor $\eta_{L}$ should be chosen such that

$$
F_{r, \Delta}(t)=F_{r, \Delta}\left(-\eta_{L} \sqrt{n}\right)=F_{r, \Delta}\left(t_{r, \Delta ; \gamma}\right)=\gamma,
$$

where $t_{r, \Delta ; \gamma}$ is the quantile of order $\gamma$ for the non-central $t$-distribution with $r$ degrees of freedom and non-centrality parameter $\Delta$. It follows from (32) that

$$
\eta_{L}=-\frac{t_{r, \Delta ; \gamma}}{\sqrt{n}}
$$

It follows from (30) that $L_{k}=\bar{X}+\eta_{L} S_{1}$. This completes the proof.

Corollary 3.1. It follows from (29) that $\operatorname{Pr}\left(\eta_{L} \sqrt{n} \sqrt{W}+V_{1} \leq z_{1-\delta_{\beta}} \sqrt{n}\right)$ can be transformed as follows:

$$
\begin{gathered}
\operatorname{Pr}\left(\eta_{L} \sqrt{n} \sqrt{W}+V_{1} \leq z_{1-\delta_{\beta}} \sqrt{n}\right)=\operatorname{Pr}\left(V_{1} \leq-\eta_{L} \sqrt{n} \sqrt{W}+z_{1-\delta_{\beta}} \sqrt{n}\right) \\
=\int_{-\infty}^{-\eta_{L} \sqrt{n} \sqrt{W}+z_{1-\delta_{\beta}} \sqrt{n}} f_{1}\left(v_{1}\right) d v_{1}=\Phi\left(-\eta_{L} \sqrt{n} \sqrt{W}+z_{1-\delta_{\beta}} \sqrt{n}\right)=\Phi(t \sqrt{W}-\Delta),
\end{gathered}
$$

where

$$
t=-\eta_{L} \sqrt{n}, \quad \Delta=-z_{1-\delta_{\beta}} \sqrt{n} .
$$

Then it follows from (25) and (34) that $t$ has to be found such that 


$$
\begin{gathered}
t=\arg (E\{\Phi(t \sqrt{W}-\Delta)\}=\gamma)=\arg \left(\int_{0}^{\infty} \Phi(t \sqrt{w}-\Delta) f_{r}(w) d w=\gamma\right) \\
=\arg \left(\frac{r^{r / 2}}{2^{r / 2} \Gamma(r / 2)} \int_{0}^{\infty} w^{r / 2-1} \exp (-r w / 2) \Phi(t \sqrt{w}-\Delta) d w=\gamma\right) \\
=\arg \left(F_{r, \Delta}(t)=\gamma\right)=t_{r, \Delta ; \gamma}
\end{gathered}
$$

where $t_{r, \Delta ; \gamma}$ is the quantile of order $\gamma$ for the non-central $t$-distribution with $r=n-1$ degrees of freedom and non-centrality parameter $\Delta$,

$$
F_{r, \Delta}(t)=\operatorname{Pr}(T \leq t)=\frac{r^{r / 2}}{2^{r / 2} \Gamma(r / 2)} \int_{0}^{\infty} w^{r / 2-1} \exp (-r w / 2) \Phi(t \sqrt{w}-\Delta) d w .
$$

is the cumulative distribution function of $T$,

$$
\begin{gathered}
f_{r, \Delta}(t)=F_{r, \Delta}^{\prime}(t)=\frac{r^{r / 2} \exp \left(-r \Delta^{2} /\left[2\left(t^{2}+r\right)\right]\right)}{\sqrt{\pi} \Gamma(r / 2) 2^{(r+1) / 2}\left(t^{2}+r\right)^{(r+1) / 2}} \\
\times \int_{0}^{\infty} w_{\bullet}^{(r-1) / 2} \exp \left(-\frac{1}{2}\left[w_{\bullet}^{1 / 2}-\frac{t \Delta}{\sqrt{t^{2}+r}}\right]^{2}\right) d w_{\bullet}, \quad-\infty<t<\infty,
\end{gathered}
$$

is the probability density function of $T$, where

$$
W_{\bullet}=W\left(t^{2}+r\right) .
$$

Corollary 3.2. If

$$
W_{. \bullet}=\frac{W\left(t^{2}+r\right)}{2},
$$

then

$$
\begin{aligned}
f_{r, \Delta}(t) & =F_{r, \Delta}^{\prime}(t)=\frac{r^{r / 2} \exp \left(-\Delta^{2} / 2\right)}{\sqrt{\pi} \Gamma(r / 2)\left(t^{2}+r\right)^{(r+1) / 2}} \int_{0}^{\infty} w_{\bullet}^{(r-1) / 2} \exp \left(-\left[w_{\bullet \bullet}-\frac{t \Delta \sqrt{2}}{\sqrt{t^{2}+r}} w_{\bullet \bullet}^{1 / 2}\right]\right) d w_{\bullet \bullet} \\
& =\frac{r^{r / 2} \exp \left(-\Delta^{2} / 2\right)}{\sqrt{\pi} \Gamma(r / 2)\left(t^{2}+r\right)^{(r+1) / 2}} \sum_{j=0}^{\infty} \frac{\Gamma((r+j+1) / 2)}{j !}\left(\frac{t \Delta \sqrt{2}}{\sqrt{t^{2}+r}}\right)^{j}, \quad-\infty<t<\infty .
\end{aligned}
$$

This form of the density function is derived in Rao [20] and appears in Searle [21]. In both Rao and Searle, $\sqrt{\pi}$ is incorrectly omitted from the denominator. It should also be noted that the central $t$ distribution is just a special case of the non-central $t$ with $\Delta=0$.

Corollary 3.3. If $k=m=1$, then

$$
\delta_{\beta}=\beta, \quad \Delta=-z_{1-\beta} \sqrt{n} .
$$

\subsection{Upper Tolerance Limit}

Theorem 4. Let $X_{1}, \ldots, X_{n}$ be observations from a preliminary sample of size $n$ from a normal distribution defined by the density function (10). Then an upper one-sided $\beta$-content tolerance limit at level $\gamma, U_{k}$ 
$\equiv U_{k}(S)$ (on the $k$ th order statistic $Y_{k}$ from a set of $m$ future ordered observations $Y_{1} \leq \ldots \leq Y_{m}$ also from the distribution (10) ), which satisfies

$$
\operatorname{Pr} P_{\theta}\left(Y_{k} \leq U_{k} \mid m\right) \geq \beta=\gamma
$$

is given by

$$
U_{k}=\bar{X}+\eta_{U} S_{1},
$$

where

$$
\eta_{U}=\frac{t_{r, \Delta ; 1-\gamma}}{\sqrt{n}}
$$

is the upper tolerance factor, $t_{r, \Delta ; 1-\gamma}$ is the quantile of order $1-\gamma$ for the non-central $t$-distribution with $r=n-1$ degrees of freedom and non-centrality parameter $\Delta=-z_{1-\delta_{1-\beta}} \sqrt{n}, z_{1-\delta_{1-\beta}}$ denotes the $1-\delta_{1-\beta}$ quantile of a standard normal distribution,

$$
\delta_{1-\beta}=\frac{(m-k+1) q_{2(m-k+1), 2 k: 1-\beta}}{(m-k+1) q_{2(m-k+1), 2 k ; 1-\beta}+k},
$$

$q_{2(m-k+1), 2 k ; 1-\beta}$ is the quantile of order $1-\beta$ for the $F$ distribution with $2(m-k+1)$ and $2 k$ degrees of freedom.

Proof. It follows from (3), (11) and (43) that

$$
\begin{aligned}
& \operatorname{Pr} P_{\theta}\left(Y_{k} \leq U_{k} \mid m\right) \geq \beta=\operatorname{Pr}\left(\int_{\frac{1-F_{\theta}\left(U_{k}\right)}{F_{\theta}\left(U_{k}\right)} \frac{2 k}{2(m-k+1)}}^{\infty} f_{2(m-k+1), 2 k}(x) d x \geq \beta\right) \\
& =\operatorname{Pr}\left(\int_{0}^{\frac{1-F_{\theta}\left(U_{k}\right)}{F_{\theta}\left(U_{k}\right)}} f_{2(m-k+1), 2 k}^{2(m-k+1)}(x) d x \leq 1-\beta\right) \\
& =\operatorname{Pr}\left(\frac{1-F_{\theta}\left(U_{k}\right)}{F_{\theta}\left(U_{k}\right)} \frac{2 k}{2(m-k+1)} \leq q_{2(m-k+1), 2 k ; 1-\beta}\right) \\
& =\operatorname{Pr}\left(F_{\theta}\left(U_{k}\right) \geq \frac{k}{k+(m-k+1) q_{2(m-k+1), 2 k ; 1-\beta}}\right) \\
& =\operatorname{Pr}\left(\frac{1}{\sigma \sqrt{2 \pi}} \int_{U_{k}}^{\infty} \exp \left(-\frac{(y-\mu)^{2}}{2 \sigma^{2}}\right) d y \leq \frac{(m-k+1) q_{2(m-k+1), 2 k ; 1-\beta}}{(m-k+1) q_{2(m-k+1), 2 k ; 1-\beta}+k}\right) \\
& =\operatorname{Pr}\left(\frac{1}{\sqrt{2 \pi}} \int_{\frac{U_{k}-\mu}{\sigma}}^{\infty} \exp \left(-\frac{z^{2}}{2}\right) d z \leq \delta_{1-\beta}\right)=\operatorname{Pr}\left(\frac{1}{\sqrt{2 \pi}} \int_{\infty}^{\frac{U_{k}-\mu}{\sigma}} \exp \left(-\frac{z^{2}}{2}\right) d z \geq 1-\delta_{1-\beta}\right)
\end{aligned}
$$




$$
\begin{gathered}
=\operatorname{Pr}\left(\frac{U_{k}-\mu}{\sigma} \geq z_{1-\delta_{1-\beta}}\right)=\operatorname{Pr}\left(\frac{U_{k}-\bar{X}+\bar{X}-\mu}{\sigma} \geq z_{1-\delta_{1-\beta}}\right) \\
=\operatorname{Pr}\left(\frac{U_{k}-\bar{X}}{S_{1}} \sqrt{n} \frac{S_{1}}{\sigma}+\frac{\sqrt{n}(\bar{X}-\mu)}{\sigma} \geq z_{1-\delta_{1-\beta}} \sqrt{n}\right)=\operatorname{Pr}\left(\eta_{U} \sqrt{n} \sqrt{W}+V_{1} \geq z_{1-\delta_{1-\beta}} \sqrt{n}\right) \\
=\operatorname{Pr}\left(\frac{V_{1}-z_{1-\delta_{1-\beta}} \sqrt{n}}{\sqrt{W}} \geq-\eta_{U} \sqrt{n}\right)=\operatorname{Pr}\left(\frac{V_{1}+\Delta}{\sqrt{W}} \geq-\eta_{U} \sqrt{n}\right)=\operatorname{Pr}\left(T \geq-\eta_{U} \sqrt{n}\right)=1-F_{r, \Delta}(t),
\end{gathered}
$$

where

$$
\eta_{U}=\frac{U_{k}-\bar{X}}{S_{1}},
$$

is the upper tolerance factor,

$$
\Delta=-z_{1-\delta_{1-\beta}} \sqrt{n}, \quad r=n-1, \quad t=-\eta_{U} \sqrt{n} .
$$

It follows from (43), (47) and (49) that the upper tolerance factor $\eta_{u}$ should be chosen such that

$$
F_{r, \Delta}(t)=F_{r, \Delta}\left(-\eta_{U} \sqrt{n}\right)=F_{r, \Delta}\left(t_{r, \Delta ; 1-\gamma},\right)=1-\gamma,
$$

where $t_{r, \Delta ; 1-\gamma}$ is the quantile of order $1-\gamma$ for the non-central $t$-distribution with $r$ degrees of freedom and non-centrality parameter $\Delta$. It follows from (50) that

$$
\eta_{U}=-\frac{t_{r, \Delta ; 1-\gamma}}{\sqrt{n}}
$$

It follows from (48) that $U_{k}=\bar{X}+\eta_{U} S_{1}$. This completes the proof.

Remark 1. It will be noted that an upper tolerance limit may be obtained from a lower tolerance limit by replacing $\beta$ by $1-\beta, \gamma$ by $1-\gamma$.

\section{Practical Example of Finding a Warranty Assessment of Image Quality}

The image quality assessment (IQA) plays a very crucial role in image and video processing. The aim is to replace human judgment of perceived image quality with a machine evaluation. A large effort has been devoted to developing IQA measures that try to mimic human perception. While many methods and models still rely on simple measures, such as the peak-siqnal-to-noise-ratio (PSNR) and the meansquared error (MSE), many others use sophisticated signal processing techniques, such as multichannel filtering [22], [23], discrete cosine transform [24], [25], multi-scale wavelet decompositions [26], [27], and Wigner-Ville distribution [28]. To date, however, it has been very difficult to find a reliable objective measure that correlates very highly with human perception [29].

Digital images are subject to a wide variety of distortions during acquisition, processing, compression, storage, transmission and reproduction, any of which may result in a degradation of visual quality. Quality measuring is needed for many applications, for example if the designer of a medical device want to decide from which device get the better results so he want to measure the quality of the images from those devices. Quality can be measured in two ways subjective and objective. The presence of blur in an image can be easily identified by the human eye but it is difficult for the computer. In practice, however, subjective evaluation is usually too inconvenient, time-consuming and expensive. The goal of research in objective image quality assessment is to develop quantitative 
Nicholas A. Nechval, Konstantin N. Nechval, and Vladimir F. Strelchonok; A New Approach to Constructing Tolerance Limits on Order Statistics in Future Samples Coming from a Normal Distribution. Advances in Image and Video Processing, Volume 4 No 3, June (2016); pp: 1-15

measures that can automatically predict perceived image quality. Quality assessment algorithms are needed to monitor the quality for real time applications. Subjective methods are impossible to implement in real time systems, so objective methods are more attracted in recent years. All these methods want to have high correlation with human perception or judgments.

Problem description. An IQA device manufacturer has the data of image quality assessment (in terms of the spearman correlation) obtained from testing $n=10$ IQA devices. These data are given in Table 1.

Table 1. The data of image quality assessment.

\begin{tabular}{|c|c|c|c|c|c|c|c|c|c|}
\hline \multicolumn{10}{|c|}{ Observations (in terms of the spearmen correlation) } \\
\hline$X_{1}$ & $X_{2}$ & $X_{3}$ & $X_{4}$ & $X_{5}$ & $X_{6}$ & $X_{7}$ & $X_{8}$ & $X_{9}$ & $X_{10}$ \\
\hline 0.913 & 0.916 & 0.923 & 0.926 & 0.936 & 0.947 & 0.961 & 0.971 & 0.975 & 0.992 \\
\hline
\end{tabular}

A buyer tells the device manufacturer that he wants to place two orders for the same type of IQA devices to be shipped to two different destinations. The buyer wants to select a random sample of $m=5$ IQA devices from each shipment to be tested. An order is accepted only if all of 5 IQA devices in each selected sample meet the warranty image quality assessment (in terms of the spearman correlation). What warranty IQA (in terms of the spearman correlation) should the manufacturer offer so that all of 5 IQA devices in each selected sample meet the warranty with probability of 0.95 ?

In order to find this warranty IQA, the manufacturer wishes to use a random sample of size $n=10$ given in Table 1 and to calculate the lower one-sided simultaneous tolerance limit $L_{k=1}(S)$ (warranty IQA) which is expected to capture a certain proportion, say, $\beta=0.95$ or more of the population of selected items $(m=5)$, with a given confidence level $\gamma=0.95$. This tolerance limit is such that one can say with a certain confidence $\gamma$ that at least $100 \beta \%$ of the IQA devices in each sample selected by the buyer for testing will give image quality assessment (in terms of the spearman correlation) no less than $L_{1}(S)$.

Goodness-of-fit testing. It is assumed that the data of Table 1 follow the normal probability distribution

$$
F_{\theta}(x)=\frac{1}{\sigma \sqrt{2 \pi}} \int_{-\infty}^{x} \exp \left(-\frac{(x-\mu)^{2}}{2 \sigma^{2}}\right) d x
$$

where the parameters $\mu$ and $\sigma$ are unknown. Thus, for the above example, we have that $n=10, m=5$, $k=1, \beta=0.95, \gamma=0.95$,

$$
S=\left(\bar{X}=\sum_{i=1}^{n} X_{i} / n=0.946, S_{1}^{2}=\sum_{i=1}^{n}\left(X_{i}-\bar{X}\right)^{2} /(n-1)=0.000758\right) .
$$

We assess the statistical significance of departures from the model (52) by performing the AndersonDarling goodness-of-fit test. The Anderson-Darling test statistic value is determined by (e.g. [30]):

$$
A^{2}=-\left[\sum_{i=1}^{n}(2 i-1)\left(\ln F_{\theta}\left(x_{i}\right)+\ln \left(1-F_{\theta}\left(x_{n+1-i}\right)\right)\right)\right] / n-n,
$$

where $F_{\theta}()$ is the cumulative distribution function ,

$$
\theta=\left(\mu=\bar{x}, \sigma=s_{1}\right)
$$

$n$ is the number of observations. 
The result from (54) needs to be modified for small sampling values. For the normal distribution the modification of $A^{2}$ is

$$
A_{\text {mod }}^{2}=A^{2}\left(1+0.75 / n+2.25 / n^{2}\right) \text {. }
$$

The $A_{\text {mod }}^{2}$ value must then be compared with critical values, $A_{\alpha}^{2}$, which depend on the significance level $\alpha$ and the distribution type. As an example, for the normal distribution the determined $A_{\bmod }^{2}$ value has to be less than the following critical values for acceptance of goodness-of-fit (see Table 2):

Table 2. Critical values for $A_{\mathrm{mod}}^{2}$.

\begin{tabular}{|c|c|c|c|c|}
\hline$\alpha$ & 0.1 & 0.05 & 0.025 & 0.01 \\
\hline$A_{\alpha}^{2}$ & 0.631 & 0.752 & 0.873 & 1.035 \\
\hline
\end{tabular}

For this example, $\alpha=0.05, A_{\alpha=0.05}^{2}=0.752$,

$$
\begin{gathered}
A^{2}=-\left[\sum_{i=1}^{10}(2 i-1)\left(\ln F_{\theta}\left(x_{i}\right)+\ln \left(1-F_{\theta}\left(x_{n+1-i}\right)\right)\right)\right] / 10-10=0.296378, \\
A_{\mathrm{mod}}^{2}=A^{2}\left(1+0.75 / 10+2.25 / 10^{2}\right)=0.325275<A_{\alpha=0.05}^{2}=0.752 .
\end{gathered}
$$

Thus, there is not evidence to rule out the normal model (52).

Finding lower tolerance limit (warranty assessment of image quality). Now the lower one-sided simultaneous $\beta$-content tolerance limit at the confidence level $\gamma, L_{1} \equiv L_{1}(S)$ (on the order statistic $Y_{1}$ from a set of $m=5$ future ordered observations $\left.\left(Y_{1} \leq \ldots \leq Y_{m}\right)\right)$ can be obtained from (26).

Since $m=5, k=1, \beta=0.95$,

$$
\begin{aligned}
& \delta_{\beta}=\frac{(m-k+1) q_{2(m-k+1), 2 k ; \beta}}{(m-k+1) q_{2(m-k+1), 2 k ; \beta}+k}=0.989796, \\
& r=n-1=9, \quad \Delta=-z_{1-\delta_{\beta}} \sqrt{n}=7.3325, \quad \gamma=0.95,
\end{aligned}
$$

the quantile of order $\gamma$ for the non-central $t$-distribution with $r$ degrees of freedom and non-centrality parameter $\Delta$ is given by

$$
t_{r, \Delta ; \gamma}=\arg \left(F_{r, \Delta}(t)=\gamma\right)=12.5512,
$$

the lower tolerance factor is given by

$$
\eta_{L}=-\frac{t_{r, \Delta ; \gamma}}{\sqrt{n}}=-3.96904,
$$

it follows from (26) that

$$
L_{k=1}=\bar{X}+\eta_{L} S_{1}=0.837 .
$$


Nicholas A. Nechval, Konstantin N. Nechval, and Vladimir F. Strelchonok; A New Approach to Constructing Tolerance Limits on Order Statistics in Future Samples Coming from a Normal Distribution. Advances in Image and Video Processing, Volume 4 No 3, June (2016); pp: 1-15

Statistical inference. Thus, the manufacturer has $95 \%$ assurance that at least $100 \beta \%$ of the IQA devices in each sample $(m=5)$ selected by the buyer for testing will give the warranty assessment of image quality (in terms of the spearman correlation) no less than $L_{1}=0.837$.

\section{Conclusion}

Tolerance limits enjoy a fairly rich history in the literature and have a very important role in engineering and manufacturing applications. In contrast to other statistical limits commonly used for statistical inference, the tolerance limits (especially for the order statistics) are used relatively rarely. One reason is that the theoretical concept and computational complexity of the tolerance limits is significantly more difficult than that of the standard confidence and prediction limits. Thus it becomes necessary to use new or innovative approaches which will allow one to construct tolerance limits on future order statistics for many populations.

\section{REFERENCES}

[1]. Mendenhall, V., A bibliography on life testing and related topics. Biometrika, 1958. XLV: p. 521-543.

[2]. Guttman, I., On the power of optimum tolerance regions when sampling from normal distributions. Annals of Mathematical Statistics, 1957. XXVIII: p. 773-778.

[3]. Wald, A. and J. Wolfowitz, Tolerance limits for a normal distribution. Annals of Mathematical Statistics, 1946. XVII: p. 208-215.

[4]. Wallis, W. A., Tolerance intervals for linear regression, in Second Berkeley Symposium on Mathematical Statistics and Probability, 1951. Berkeley: University of California Press, p. 43-51.

[5]. Patel, J. K., Tolerance limits: a review. Communications in Statistics: Theory and Methodology, 1986. 15: p. 2719-2762.

[6]. Dunsmore, I. R., Some approximations for tolerance factors for the two parameter exponential distribution. Technometrics, 1978. 20: p. 317-318.

[7]. Guenther, W. C., S. A. Patil, and V. R. R. Uppuluri, One-sided $\beta$-content tolerance factors for the two parameter exponential distribution. Technometrics, 1976. 18: p. 333-340.

[8]. Engelhardt, M. and L. J. Bain, Tolerance limits and confidence limits on reliability for the twoparameter exponential distribution. Technometrics, 1978. 20: p. 37-39.

[9]. Guenther, W. C., Tolerance intervals for univariate distributions. Naval Research Logistics Quarterly, 1972. 19: p. 309-333.

[10]. Hahn, G. J. and W. Q. Meeker, Statistical Intervals: A Guide for Practitioners. 1991. New York: John Wiley \& Sons.

[11]. Nechval, N. A. and K. N. Nechval, Tolerance limits on order statistics in future samples coming from the two-parameter exponential distribution. American Journal of Theoretical and Applied Statistics, 2016. 5: p. 1-6.

[12]. Johnson, N. L., S. Kotz, and N. Balakrishnan, Continuous Univariate Distributions, vol. 1. Second edition, 1994. New York: John Wiley \& Sons. 
[13]. Patel, J. K. and C. B. Read, Handbook of the Normal Distribution. Second edition, 1996. New York: Marcel Dekker.

[14]. Balakrishnan, N. and V. B. Nevzorov, A Primer on Statistical Distributions. 2003. New Jersey: Wiley.

[15]. Kotz, S. and D. Vicari, Survey of developments in the theory of continuous skewed distributions. Metron, 2005. 63: p. 225-261.

[16]. Nechval, N. A. and E. K. Vasermanis, Improved Decisions in Statistics. 2004. Riga: Izglitibas soli.

[17]. Nechval, N. A., K. N. Nechval, and E. K. Vasermanis, Effective state estimation of stochastic systems. Kybernetes (An International Journal of Systems \& Cybernetics), 2003. 32: p. 666-678.

[18]. Nechval, N. A., G. Berzins, M. Purgailis, and K. N. Nechval, Improved estimation of state of stochastic systems via invariant embedding technique. WSEAS Transactions on Mathematics, 2008. 7: p. 141159.

[19]. Nechval, N. A., M. Purgailis, K. N. Nechval, and V. F. Strelchonok, Optimal predictive inferences for future order statistics via a specific loss function. IAENG International Journal of Applied Mathematics, 2012. 42: p. 40-51.

[20]. Rao, C. R., Linear Statistical Inference and its Applications. 1965. New York: Wiley.

[21]. Searle, S. R., Linear Models. 1971. New York: Wiley.

[22]. Daly S., The visual difference predictor: an algorithm for the assessment of image fidelity, in Human Vision, Visual Processing, and Digital Display, Proc. SPIE, vol. 1666, 1992, San Jose, CA, p. 2-15.

[23]. Heeger, D. J. and P. C. Teo, A model of perceptual image fidelity, in Proc. IEEE International Conference on Image Processing, vol. 2, 1995, p. 343-345.

[24]. Malo, J., A. M. Pons, and 5. M. Artigas, Subjective image fidelity metric based on bit allocation of the human visual system in the DCT domain. Image und Vision Computing, 1997. 15: p. 535-548.

[25]. Watson, A. B., J. Hu, and J. F. McGowm, Digital video quality metric based on human vision. J. of Electronic Imaging, 2001. 10: p. 20-29.

[26]. Lai, Y. K. and C.-C. J. Kuo, A Haar wavelet approach to compressed image quality measurement. J. Visual Communication und Image Repres., 2000. 11: p. 17-40.

[27]. Beghdadi, A. and B. Pesquet-Popescu, A new image distortion measure based on wavelet decomposition, in Proc. Seventh Intern. Symp. Signal Proces. its Applications (ISSPA-2003), vol. 1, 2003, Paris, p. 485-488.

[28]. Beghdadi, A. and R. Iordache, Image quality assessment using the joint space/spatial-frequency representation. EURASIP Journal on Applied Signal Processing, 2006. 6: p. 1-8.

[29]. Wang, Z., A. C. Bovik, and L. Lu, Why is image quality assessment so difficult? In Proc. IEEE Inter. Conference Acoustics, Speech, and Signal Processing (ICASSP-2002), vol. 4, 2002, Orlando FL, p. 3313-3336.

[30]. Dodson, B., Weibull Analysis. 1994. Milwaukee: ASQ Quality Press. 